Proceedings of the 2011 Winter Simulation Conference

S. Jain, R. R. Creasey, J. Himmelspach, K. P. White, and M. Fu, eds.

\title{
BROWNIAN BRIDGE HYPOTHESIS TESTING FOR THE INITIAL TRANSIENT PROBLEM
}

\author{
Peter W. Glynn \\ Management Science and Engineering \\ Stanford University \\ Stanford, CA 94305 USA
}

\author{
Eunji Lim \\ Industrial Engineering \\ University of Miami \\ Coral Gables, FL 33124 USA
}

\begin{abstract}
This paper models the detection of the initial transient in a steady-state simulation problem as a change point hypothesis testing problem. We introduce two new hypothesis tests for the initial transient, each of which is based on the Brownian bridge process and each of which is a composite test that involves testing against infinitely many alternatives (that depend on the duration of the transient period). One of our two procedures is closely related to the class of tests proposed by Schruben, Singh, and Tierney (1983).
\end{abstract}

\section{INTRODUCTION}

Let $X=(X(t): t \geq 0)$ be a real-valued stochastic process representing the output of a simulation. We assume that there exists a (deterministic) constant $\alpha$ for which

$$
\bar{X}(t)=\frac{1}{t} \int_{0}^{t} X(s) d s \Rightarrow \alpha
$$

as $t \rightarrow \infty$ (where $\Rightarrow$ denotes weak convergence).

The steady-state simulation problem is concerned with the estimation of the steady-state mean $\alpha$. In the typical systems simulation context, the simulation of $X$ is initialized with an initial distribution that is atypical of equilibrium behavior, thereby inducing a so-called initial transient period during which the system "warms up" until its dynamics are typical of steady-state. In this paper, our goal is to identify the presence of a statistically significant initial transient via use of a suitably defined hypothesis testing procedure. In particular, we view the presence of an initial transient as one in which a change point, associated with a change in the mean, is present. As noted by Schruben, Singh, and Tierney (1983), the Brownian bridge process arises naturally in this setting. Consequently, the tests arise in connection with change point problems associated with the Brownian bridge process. In contrast to Schruben et al. (1983), our tests are composite tests that test simultaneously against all possible times for the change point (thereby modeling our uncertainty in the duration of the initial transient).

This paper is organized as follows. In Section 2, we review the basis change point problem and point out its connection to the initial transient detection problem. Section 3 introduces our two new tests, both involving the Brownian bridge, while Section 4 makes the connection to the work of Schruben et al. (1983). Section 5 provides some concluding comments.

\section{VIEWING THE INITIAL TRANSIENT DETECTION PROBLEM AS A CHANGE POINT PROBLEM}

In order that $X$ satisfy the law of large numbers (1) (so that the steady-state simulation problem is welldefined), $X$ must typically "mix", so that observations collected at times that are widely separated are effectively independent of one another. When processes mix, it is generally the case that $X$ also satisfies a functional central limit theorem (FCLT) version of (1), so that there exists a (deterministic) positive 


\section{Glynn and Lim}

constant $\sigma$ for which

$$
Z_{t} \Rightarrow \sigma B
$$

as $t \rightarrow \infty$ in $D[0, \infty)$, where $B=(B(r): r \geq 0)$ is a standard Brownian motion and

$$
Z_{t}(r)=t^{-1 / 2}\left(\int_{0}^{r t} X(s) d s-r t \alpha\right)
$$

The notion of weak convergence in $D[0, \infty)$ is discussed in Ethier and Kurtz (1986), as are various limit theorems supporting (2).

In this paper, we take the view that there is an interval $\left[0, t_{0}\right]$ over which

$$
\mathbb{E} X(s)=\alpha+\delta
$$

for $0 \leq s \leq t_{0}$, after which the process $X$ is in stationarity, so that

$$
\mathbb{E} X(s)=\alpha .
$$

for $s \geq t_{0}$. In view of (2), this leads us to approximate $X$ via

$$
\int_{0}^{r} X(s) d s \stackrel{\mathscr{D}}{\approx} \alpha t+\delta\left(r \wedge t_{0}\right)+\sigma B(r)
$$

where $\stackrel{\mathscr{D}}{\approx}$ denotes "has approximately the same distribution as" (and has no rigorous meaning, other than that imposed by (2)), and $a \wedge b \triangleq \min (a, b)$ for $a, b \geq 0$. The statistical model (3) is one in which the Brownian motion exhibits a change point at time $t_{0}$, at which time its drift changes from $\alpha+\delta$ to $\alpha$. The Neyman-Pearson lemma states that the most powerful test for such a Brownian change point problem involves rejecting the hypothesis of stationarity over $[0, t]$ in favor of an initial transient of magnitude $\delta$ over $\left[0, t_{0}\right]$ (followed by stationarity over $\left[t_{0}, t\right]$ ) whenever

$$
\frac{\delta}{\sigma^{2}}\left(\int_{0}^{t_{0}} X(s) d s-\alpha t_{0}\right)-\frac{\delta^{2} t_{0}}{2 \sigma^{2}}
$$

exceeds some critical value. Since $t_{0}$ is unknown, it seems statistically reasonable to reject the hypothesis of stationarity whenever

$$
\max _{0 \leq s \leq t} \quad\left[\frac{\delta}{\sigma^{2}}\left(\int_{0}^{s} X(u) d u-\alpha s\right)-\frac{\delta^{2} s}{2 \sigma^{2}}\right]
$$

exceeds some appropriately determined value, and to estimate the end of the initial transient period $t_{0}$ as the maximizer $\widehat{s}$ of (4). Of course, if one's intuition is that the initial transient terminated sometime prior to $\beta$ (with $\beta$ unknown), one would modify the above change point test statistic to

$$
\max _{0 \leq s \leq \beta} \quad\left[\frac{\delta}{\sigma^{2}}\left(\int_{0}^{s} X(u) d u-\alpha s\right)-\frac{\delta^{2} s}{2 \sigma^{2}}\right] .
$$

The difficulty with this change point statistic is that the steady-state mean is assumed to be known to the simulationist (as is the time-average variance constant (TAVC) $\sigma^{2}$ ). Note that $\sigma^{2}$ can always be computed exactly (with probability one) from the quadratic variation of the Brownian path. Thus, the key assumption underlying this Brownian formulation of the change point problem is that the drift of the Brownian motion 


\section{Glynn and Lim}

is known either prior or subsequent to the change point; see Page (1954) and Pollak and Siegmund (1985) for two representative papers that discuss this particular formulation of the change point problem.

Note that the natural estimator for $\alpha$ is $\bar{X}(t)$, thereby leading to consideration of a test statistic based on

$$
\begin{aligned}
\frac{\delta}{\sigma^{2}} & \left(\int_{0}^{s} X(u) d u-s \bar{X}(t)\right) \\
& =\frac{\delta}{\sigma^{2}}\left(\int_{0}^{s} X(u) d u-\frac{s}{t} \int_{0}^{t} X(u) d u\right) \\
& \stackrel{\mathscr{D}}{\approx} \frac{\delta}{\sigma^{2}}(\sigma B(s)-s \sigma(B(t) / t)) \\
& =\frac{\delta}{\sigma^{2}}(\sigma B(r t)-r \sigma B(t)) \quad(r=s / t) \\
& \stackrel{\mathscr{D}}{=} \sqrt{t}(\delta / \sigma)(B(r)-r B(1)),
\end{aligned}
$$

where $\stackrel{\mathscr{D}}{=}$ denotes equality in distribution, and the scaling self-similarity relationship $B(\cdot t) \stackrel{\mathscr{D}}{=} t^{1 / 2} B(\cdot)$ has been used for the last equality. This leads to consideration of change point tests based on the so-called (standard) Brownian bridge process $B_{0}=\left(B_{0}(t): 0 \leq t \leq 1\right)$ defined by

$$
B_{0}(t)=B(t)-t B(1) \text {. }
$$

In the next section, we discuss change point tests that are based on a Brownian bridge formulation.

\section{HYPOTHESIS TESTING BASED ON THE BROWNIAN BRIDGE}

The connection between the Brownian bridge process and hypothesis testing for the initial transient problem was first made by Schruben, Singh, and Tierney (1983). We discuss their proposed test in detail in Section 4. To derive appropriate hypothesis tests in this setting, note first that if $X$ is in equilibrium over the entire simulated time horizon $[0, t]$, then

$$
\begin{aligned}
\int_{0}^{t_{0}} X(u) d u-t_{0} \bar{X}(t) \\
=\frac{\left(t-t_{0}\right)}{t} \int_{0}^{t_{0}} X(u) d u-\frac{t_{0}}{t} \int_{t_{0}}^{t} X(u) d u \\
\quad \stackrel{\mathscr{D}}{\approx} \frac{\left(t-t_{0}\right)}{t} \sigma B\left(t_{0}\right)-\frac{t_{0}}{t} \sigma\left(B(t)-B\left(t_{0}\right)\right) \\
\stackrel{\mathscr{D}}{=} \sqrt{\left(1-t_{0} / t\right) t_{0}} \sigma N(0,1),
\end{aligned}
$$

where $N(0,1)$ is a standard normal random variable having mean 0 and variance 1 . On the other hand, if $X$ exhibits a change point from mean $\alpha+\delta$ over $\left[0, t_{0}\right]$ to $\alpha$ over $\left[t_{0}, t\right]$, then

$$
\begin{aligned}
& \int_{0}^{t_{0}} X(u) d u-t_{0} \bar{X}(t) \\
& \quad \stackrel{\mathscr{D}}{\approx} \delta t_{0}\left(1-t_{0} / t\right)+\sqrt{\left(1-t_{0} / t\right) t_{0}} \sigma N(0,1) .
\end{aligned}
$$

As a consequence, the ratio of the likelihood of the data under a change point at $t_{0}$ from mean $\alpha+\delta$ to mean $\alpha$ relative to the likelihood of no change point (i.e. no statistically significant initial transient) over $[0, t]$ is approximately

$$
\exp \left(\frac{\delta}{\sigma^{2}}\left(\int_{0}^{t_{0}} X(u) d u-t_{0} \bar{X}(t)\right)-\frac{\delta^{2}}{2 \sigma^{2}} t_{0}\left(1-t_{0} / t\right)\right)
$$




\section{Glynn and Lim}

Thus, the Neyman-Pearson lemma asserts that an approximately optimal hypothesis test takes the form: Reject the hypothesis of stationarity over $[0, t]$ (in favor of a change point at $t_{0}$ ) if

$$
\frac{\delta}{\sigma^{2}}\left(\int_{0}^{t_{0}} X(u) d u-t_{0} \bar{X}(t)\right)-\frac{\delta^{2}}{2 \sigma^{2}} t_{0}\left(1-t_{0} / t\right)>\widetilde{z},
$$

where $\widehat{z}$ is chosen so that under the assumption of stationarity (the so-called "null-hypothesis" in this setting), the Type 1 error is no more than (say) $\gamma$. Given the FCLT (2), this amounts to choosing $\widehat{z}$ so that

$$
\mathbb{P}\left(\left(\frac{\delta t^{1 / 2}}{\sigma}\right) B_{0}(r)-\frac{1}{2}\left(\frac{\delta t^{1 / 2}}{\sigma}\right)^{2} r(1-r)>\widetilde{z}\right)=\gamma,
$$

where $r=t_{0} / t$. This formula makes clear that a key test parameter is $\delta t^{1 / 2} / \sigma$. In particular, the power of the test is largely determined by $\delta t^{1 / 2} / \sigma$, so that the larger this quantity, the greater the power of the test to accurately detect the presence of the alternative hypothesis (i.e. the presence of an initial transient ending at $t_{0}$ ). As a consequence, $\delta$ can be chosen in proportion to $\sigma / t^{1 / 2}$ without affecting the power of the test. It should further be noted that the test based on (6) is uniformly most powerful given all alternatives satisfying $\delta^{\prime} \geq \delta$.

As in Section 2, the time $t_{0}$ of the change point is unknown. If one's intuition is that the initial transient must terminate prior to deterministic time $\beta \leq t$, this suggests consideration of the test statistic

$$
\max _{0 \leq s \leq \beta} \quad\left[\frac{\delta}{\sigma^{2}}\left(\int_{0}^{s} X(u) d u-s \bar{X}(t)\right)-\frac{\delta^{2} s}{2 \sigma^{2}}(1-s / t)\right] .
$$

The obvious two-sided alternative (testing for initial transients of magnitude $\delta$ both above and below $\alpha)$ is then

$$
\max _{0 \leq s \leq \beta} \quad\left[\left|\frac{\delta}{\sigma^{2}}\left(\int_{0}^{s} X(u) d u-s \bar{X}(t)\right)\right|-\frac{\delta^{2} s}{2 \sigma^{2}}(1-s / t)\right] .
$$

The above testing procedure requires specifying $\delta$. One means of avoiding this is to consider $\delta$ as a parameter and to estimate $\delta$ from (5) via the principle of maximum likelihood, thereby leading to the estimator

$$
\widehat{\delta}=\frac{\int_{0}^{t_{0}} X(u) d u-t_{0} \bar{X}(t)}{t_{0}\left(1-t_{0} / t\right)} .
$$

If we substitute this estimator into the test procedure (6), maximize over $s \in[0, \beta]$, and take the square root, we are led to consideration of the test statistic

$$
\frac{1}{\sigma} \max _{0 \leq s \leq \beta} \frac{\left|\int_{0}^{s} X(u) d u-s \bar{X}(t)\right|}{\sqrt{s(1-s / t)}} .
$$

Both of the test statistics (7) and (9) are considered in James, James, and Siegmund (1987), as are several other possibilities. In view of (2), the critical values $z_{1}$ and $z_{2}$ corresponding to (8) and (9) are respectively determined by the equations

$$
\mathbb{P}\left(\max _{0 \leq r \leq \beta / t}\left(B_{0}(r)-\frac{\delta}{2 \sigma} \sqrt{t} r(1-r)\right)>\frac{z_{1} \sigma}{\delta \sqrt{t}}\right)=\gamma
$$

and

$$
\mathbb{P}\left(\max _{0 \leq r \leq \beta / t} \frac{\left|B_{0}(r)\right|}{\sqrt{r(1-r)}}>z_{2} \sigma\right)=\gamma
$$




\section{Glynn and Lim}

for a given level $\gamma$ of Type 1 error. In practice, the TAVC $\sigma^{2}$ is unknown and must be estimated from the observed data. So long as $\hat{\sigma}_{t}^{2}$ is a consistent estimator for $\sigma^{2}$ (i.e. $\widehat{\sigma}_{t}^{2} \Rightarrow \sigma^{2}$ as $t \rightarrow \infty$ ), substitution of $\widehat{\sigma}_{t}$ for $\sigma$ in our testing procedures is asymptotically permissible. The tests based on (8) and (9) are our proposed (new) tests for detecting the presence of the initial transient. Approximations to the critical values $z_{1}$ and $z_{2}$ can be found in James et al. (1987).

\section{THE SCHRUBEN-SINGH-TIERNEY INITIALIZATION TEST}

As noted earlier, it is natural to consider the process

$$
Y_{t}(s)=\int_{0}^{s} X(u) d u-s \bar{X}(t), \quad 0 \leq s \leq t
$$

when the steady-state mean $\alpha$ is unknown. According to (2), when $X$ is in stationarity over $[0, t]$,

$$
\begin{aligned}
Y_{t}(s) & \stackrel{\mathscr{D}}{\approx} \sigma B(s)-s \sigma B(t) / t \\
& \stackrel{\mathscr{D}}{=} \sigma \sqrt{t}(B(s / t)-(s / t) B(1)) \\
& =\sigma \sqrt{t} B_{0}(s / t),
\end{aligned}
$$

so

$$
Y_{t}(r t) \stackrel{\mathscr{D}}{\sigma} \sigma \sqrt{t} B_{0}(r), \quad 0 \leq r \leq 1
$$

In other words, under the assumption of stationarity,

$$
\frac{Y_{t}(r t)}{\sigma \sqrt{t}} \stackrel{\mathscr{D}}{\approx} B_{0}(r), \quad 0 \leq r \leq 1
$$

On the other hand, if we model the initial transient as a (possibly) non-constant drift $\mu(\cdot)$ added to an equilibrium version $X^{*}$ of $X$, then

$$
\begin{aligned}
\frac{Y_{t}(r t)}{\sigma \sqrt{t}} & =\frac{\int_{0}^{r t} X^{*}(u) d u-r \int_{0}^{t} X^{*}(u) d u}{\sigma \sqrt{t}}+\frac{\int_{0}^{r t} \mu(u) d u-r \int_{0}^{t} \mu(u) d u}{\sigma \sqrt{t}} \\
& \stackrel{\mathscr{D}}{\approx} B_{0}(r)+\int_{0}^{r} \widetilde{\mu}_{t}(v) d v
\end{aligned}
$$

for $0 \leq r \leq 1$, where

$$
\widetilde{\mu}_{t}(v)=\frac{t^{1 / 2}}{\sigma}\left[\mu(v t)-\int_{0}^{1} \mu(s t) d s\right] .
$$

In contrast to the calculations of Section 3, Schruben et al. (1983) consider the likelihood of the entire path of $Y_{t}(\cdot)$ in developing their hypothesis test (to be contrasted against Section 3's calculation at a given fixed $t_{0}$ ). Following the discrete-time approach of Schruben et al. (1983), we find that the logarithm of the likelihood of the path under the initial transient model $\mu(\cdot)$ to that computed under the assumption of stationarity is

$$
\begin{aligned}
& \int_{0}^{1} \widehat{\mu}_{t}(r) \frac{d Y_{t}(r)}{\sigma \sqrt{t}}-\frac{1}{2} \int_{0}^{1} \widehat{\mu}_{t}(r)^{2} d r \\
& =\frac{1}{\sigma^{2}} \int_{0}^{1}\left[\mu(r t)-\int_{0}^{1} \mu(s t) d s\right](t X(r t)-t \bar{X}(t)) d r-\frac{1}{2 \sigma^{2}} \int_{0}^{1}\left[\mu(r t)-\int_{0}^{1} \mu(s t) d s\right]^{2} t d r \\
& =\frac{1}{\sigma^{2}} \int_{0}^{t}\left[\mu(v)-\frac{1}{t} \int_{0}^{t} \mu(l) d l\right](X(v)-\bar{X}(t)) d v-\frac{1}{2 \sigma^{2}} \int_{0}^{t}\left[\mu(v)-\frac{1}{t} \int_{0}^{t} \mu(l) d l\right]^{2} d v
\end{aligned}
$$




\section{Glynn and Lim}

In particular, when we presume (as in Section 3) that $\mu(l)=\delta$ for $1 \leq l \leq s$ and is zero for $l>s$, then the $\log$-likelihood is

$$
\frac{\delta}{\sigma^{2}}\left(\int_{0}^{s} X(r) d r-s \bar{X}(t)\right)-\frac{\delta^{2}}{2 \sigma^{2}}(1-s / t),
$$

which matches (6). Maximizing over $s$ then recovers the test (7). Thus, the first of our two hypothesis tests (7) and (9) of Section 3 can be viewed as a composite version of the Schruben et al. (1983) test, in which we simultaneously test against all alternatives that involve a constant drift over an interval $[0, s](s \leq \beta)$, followed by stationarity subsequent to time $s$. The second test, based on (9), is also a composite test, and does not appear to have any direct relation to the tests proposed by Schruben et al. (1983) (other than the fact that it is also based on a Brownian bridge analysis).

\section{FURTHER WORK}

One key issue that needs to be addressed is the empirical performance of our two proposed composite tests, as compared to those introduced in Schruben et al. (1983), as well as relative to additional initial transient tests suggested in the years since. In future work, we expect to perform such an evaluation, focusing on settings in which the initial transient can significantly impair the performance of the steady-state estimator $\bar{X}(t)$. In addition, we will consider additional such tests, in which the initial transient is described by a more complex model than that of a simple change point in which the mean is constant over the initial transient period. The intention is to develop tests that are distributionally robust, yet sensitive enough to pick up significant departures from stationarity.

\section{REFERENCES}

Ethier, S. N., and T. G. Kurtz. 1986. Markov Processes: Characterization and Convergence. New York: Wiley.

James, B., K. L. James, and D. Siegmund. 1987. "Tests for a change-point”. Biometrika 74:71-83.

Page, E. S. 1954. "Continuous inspection schemes". Biometrika 41:100-115.

Pollak, M., and D. Siegmund. 1985. "A diffusion process and its applications to detecting a change in the drift of Brownian motion". Biometrika 72:267-280.

Schruben, L., H. Singh, and L. Tierney. 1983. "Optimal tests for initialization bias in simulation output". Operations Research 31:1167-1178.

\section{AUTHOR BIOGRAPHIES}

PETER W. GLYNN is the current Chair of the Department of Management Science and Engineering at Stanford University. He received his Ph.D in Operations Research from Stanford University in 1982. He then joined the faculty of the University of Wisconsin at Madison, where he held a joint appointment between the Industrial Engineering Department and Mathematics Research Center, and courtesy appointments in Computer Science and Mathematics. In 1987, he returned to Stanford, where he joined the Department of Operations Research. He is now the Thomas Ford Professor of Engineering in the Department of Management Science and Engineering, and also holds a courtesy appointment in the Department of Electrical Engineering. From 1999 to 2005, he served as Deputy Chair of the Department of Management Science and Engineering, and was Director of Stanford's Institute for Computational and Mathematical Engineering from 2006 until 2010. He is a Fellow of INFORMS and a Fellow of the Institute of Mathematical Statistics, has been co-winner of Best Publication Awards from the INFORMS Simulation Society in 1993 and 2008, was a co-winner of the Best (Biannual) Publication Award from the INFORMS Applied Probability Society in 2009, and was the co-winner of the John von Neumann Theory Prize from INFORMS in 2010. His research interests lie in simulation, computational probability, queueing theory, statistical inference for stochastic processes, and stochastic modeling. His e-mail address is glynn@stanford.edu. 


\section{Glynn and Lim}

EUNJI LIM is an Assistant Professor in the Department of Industrial Engineering at the University of Miami. Her research interest includes stochastic optimization and simulation. Her e-mail address is lim@ miami.edu. 